\title{
Validation of Vibro-Impact Force Models by Numerical Simulation, Perturbation Methods and Experiments
}

de Souza Reboucas, Geraldo F.; Santos, IImar F.; Thomsen, Jon J.

Published in:

Journal of Sound and Vibration

Link to article, DOI:

10.1016/j.jsv.2017.10.028

Publication date:

2018

Document Version

Peer reviewed version

Link back to DTU Orbit

Citation (APA):

de Souza Reboucas, G. F., Santos, I. F., \& Thomsen, J. J. (2018). Validation of Vibro-Impact Force Models by Numerical Simulation, Perturbation Methods and Experiments. Journal of Sound and Vibration, 413, 291-307. https://doi.org/10.1016/j.jsv.2017.10.028

\section{General rights}

Copyright and moral rights for the publications made accessible in the public portal are retained by the authors and/or other copyright owners and it is a condition of accessing publications that users recognise and abide by the legal requirements associated with these rights.

- Users may download and print one copy of any publication from the public portal for the purpose of private study or research.

- You may not further distribute the material or use it for any profit-making activity or commercial gain

- You may freely distribute the URL identifying the publication in the public portal 


\title{
Validation of Vibro-Impact Force Models by Numerical Simulation, Perturbation Methods and Experiments
}

\author{
Geraldo F. de S. Rebouças ${ }^{\mathrm{a}}$, Ilmar F. Santos ${ }^{\mathrm{a}, *}$, Jon J. Thomsen ${ }^{\mathrm{a}}$ \\ ${ }^{a}$ Department of Mechanical Engineering, Technical University of Denmark - DTU, Denmark
}

\begin{abstract}
The frequency response of a single degree of freedom vibro-impact oscillator is analyzed using Harmonic Linearization, Averaging and Numeric Simulation, considering three different impact force models: one given by a piecewise-linear function (Kelvin-Voigt model), another by a high-order power function, and a third one combining the advantages of the other two. Experimental validation is carried out using control-based continuation to obtain the experimental frequency response, including its unstable branch.

Keywords: vibro-impact dynamics ; impact force models ; Kelvin-Voigt impact

model ; Power-law impact model ; vibro-impacting beam ; lumped mass beam ; experimental validation ; control-based continuation ;
\end{abstract}

\section{Introduction}


The contact between two bodies can be modeled as a temporary association of the stiffness and damping properties of the colliding bodies, each one modeled as a linear system. Despite its simplicity, this approach has some limitations, mainly the nonzero values of the impact force on the initial and final parts of the contact phase due to dissipative forces. This motivated the development of other contact models such as the one suggested by Hunt and Crossley [4], who derived the damping coefficient as a power function of the impact deformation. This solved the physical inaccuracy of the linear model and lead to an ongoing discussion about the appropriate form of the nonlinear dissipative term [5, 6]. Most of the expressions for the dissipative term combine the velocity immediately before impact and the coefficient of restitution with a rational power of the contact deformation, leading to complicated expressions whose analysis is possible only through numerical simulation.

One of the oldest techniques to model impacting systems is to use a coefficient of restitution $(\mathrm{CoR})$ to relate the velocities before and after impact. This classic approach has been used in many applications [7-[11]. For instance, Bishop et al. [7] used a single degree of freedom (SDOF) oscillator with a CoR rule to reproduce the experimental behavior of an impacting cantilever beam around its first natural frequency. Also, the coefficient of restitution, together with a power-law elastic force, can be used to obtain various nonlinear contact force models, such as the one mentioned previously by Hunt and Crossley [4]. Despite its popularity, it should be pointed out that the CoR is not an intrinsic property of the material, depending on the impact velocity [12]. Also, using a simple CoR kinematic rule does not give any direct information about contact forces or stresses.

From the numerical point of view, one can replace discontinuities by smooth equivalent functions and use standard techniques to solve the smoothed model as done by Savi et al. in [13], where the impact condition is smoothed, or by Elmegård et al. [14], who applied numeric continuation to a smoothed model of a lumped-mass impacting beam. In these cases, while it is safer to use standard ODE solvers provided by accredited sources, it is also necessary to properly tune smooth approximations of discontinuous functions; see [15] for a discussion on the effect of smoothing functions on the frequency response of oscillators with clearance. There are also several numerical 
integration algorithms designed for non-smooth systems [16-18], but their comparison is out of the scope of this work.

Vibro-impact oscillators can also be analysed using common perturbation techniques, such as Harmonic Linearization [19, 20], Averaging [21--23], and the LindstedtPoincaré method [24]. Besides their widespread use with nonlinear problems, these methods assume weak and smooth nonlinearities, which are not reasonable assumptions for general impacting systems. This limitation motivated the development of non-smooth transformations with respect to state [25, 26] and time [27] variables. By these, one can remove non-smooth terms from a certain model, or make them small, allowing the usage of common perturbation methods. An example of such combination can be found in [8] where non-smooth transformations were used to weaken a near-elastic kinematic impact condition, enabling subsequent application of extended averaging.

In some cases, the applicability of the theoretical/numerical tools mentioned above is accompanied by physical experiments. In [28] the experimental chaotic behavior of a base-excited cantilever beam with one-sided stop is qualitatively compared with results from numeric simulations of a piecewise linear oscillator. In [7] the experimental frequency response of a forced cantilever beam with a unilateral constraint is compared with the one obtained by numeric simulation of a SDOF oscillator with a coefficient of restitution, showing reasonable accuracy. The Averaging method has been extensively used to obtain analytical frequency-amplitude expressions for piecewise linear oscillators with one [21, 22] and two [23] degrees of freedom.

In [13] the nonlinear dynamics of a mass-spring system with discontinuous stiffness and damping was analyzed experimentally and numerically by smoothing the impact condition. A similar numerical-experimental analysis was performed by Aguiar and Weber [29], focusing on the behavior of the impact force. Using control-based continuation Bureau et al. [30] obtained experimental frequency responses of a cantilever beam with lumped mass and bilateral constraints. A single-DOF numerical model for this system was proposed by Elmegård et al. [14], who used the experimental data from [30] to validate the model and predict the existence of an isola, which was later identified experimentally by Bureau et al. [31]. 
From the overview presented above, one can realize that besides the match between results from particular numerical/analytical techniques with experimental findings there are little efforts on comparing the different paradigms used to model and analyze vibro-impact systems.

The objective and main originality of the present work is to compare different impact force models using analytical, numerical and experimental techniques. The frequency response of a SDOF vibro-impact oscillator is analyzed using Harmonic Linearization and Averaging, considering three different impact force models: one given by the Kelvin-Voigt model (piecewise-linear function), another using a powerlaw function and a third one combining the strengths of the first two. Experimentally, control-based continuation [30, 31] is used to obtain frequency responses of an impacting beam, including its unstable branch. Numerical simulations are used to validate the simple analytic approximations obtained by perturbation methods.

Despite the ability of the mentioned models to produce different qualitative behaviors such as quasiperiodicity and chaos, the analysis presented here is restricted to single-periodic oscillations only.

As the main contribution of this manuscript is to compare different impact force formulations, only the most common models are considered. That is the case of the Kelvin-Voigt model, which is widely used despite its inaccuracies [2, 5, 23]. The Power-law model can be viewed as a generic version of the compliant force models first derived by Hunt and Crossley [4] and further developed by others [5, 32].

\section{Experimental setup and procedure}

The experimental setup has been described previously in [30, 31] and is shown in Fig. 1. In Fig. 11, b an electrodynamic shaker (1) [B\& $\left.\mathrm{K}^{\circledR} 4808\right]$ is used to apply a harmonic excitation to a platform (2), containing a cantilever beam and a pair of symmetrically located stops (5) to restrain the lateral movement of the beam. The shaker is driven by a power amplifier [ $\left.\mathrm{B} \& \mathrm{~K}^{\circledR} 2712\right]$ and is connected to the platform by a stinger. The impacting beam can be seen in detail in Fig. 11,d, where two DC holding electromagnetic actuators (6) [Magnet-Schultz ${ }^{\circledR}$ G MH X 030] are placed on each side of the 
lumped mass (4) to execute control-based continuation. The displacement of both platform and lumped mass are measured by two laser sensors (3) [OMRON ${ }^{\circledR}$ ZX-LD40]. Due to the electromagnetic actuators around the lumped mass, its displacement is measured below the mass location, Fig. 11. A dSPACE ${ }^{\circledR}$ DS1104 R\&D controller board is used to perform data acquisition and control-based continuation of the experimental setup.

The experimental frequency response of the impacting beam, shown in Fig. 2 a, was obtained by the authors, who repeated some of the experiments done by [30, 31] using control-based continuation. In this model-free approach, the equilibrium states are found by a predictor-corrector algorithm. A non-invasive proportional-derivative controller is used to stabilize the system through its unstable branch (lower part of the bent peak) using the electromagnetic actuators (6).

Figure $2 \mathrm{p}$ illustrates the harmonic decomposition of the experimental frequency response from its static component, represented by $0 \tilde{\Omega}$, up to five times the excitation frequency $\tilde{\Omega}$. In this figure one can see that the impacting regime increases the amplitude of higher harmonics but they are still negligible if compared with the fundamental harmonic, $1 \tilde{\Omega}$.

In this work, the experimental observations are restricted to the neighborhood of the system's fundamental linear natural frequency, measured as $f_{n}=7.6 \mathrm{~Hz}$, which is much smaller than its second linear natural frequency, which is around $200 \mathrm{~Hz}$. Also, the lumped mass ( $m=0.2 \mathrm{~kg}$ ) is approximately 8 times heavier than the beam's mass, being the dominant inertial element. In addition to that, the lumped mass is located at a reasonable distance from the stops, which can be considered as rigid supports, causing inelastic impacts.

Based on the above, one can simplify the mathematical modeling of the impacting beam shown in Fig. 1 in the neighborhood of its fundamental linear natural frequency, by using a SDOF model, where the lumped mass dynamics dominate the oscillations in comparison to the flexible beam alone. 


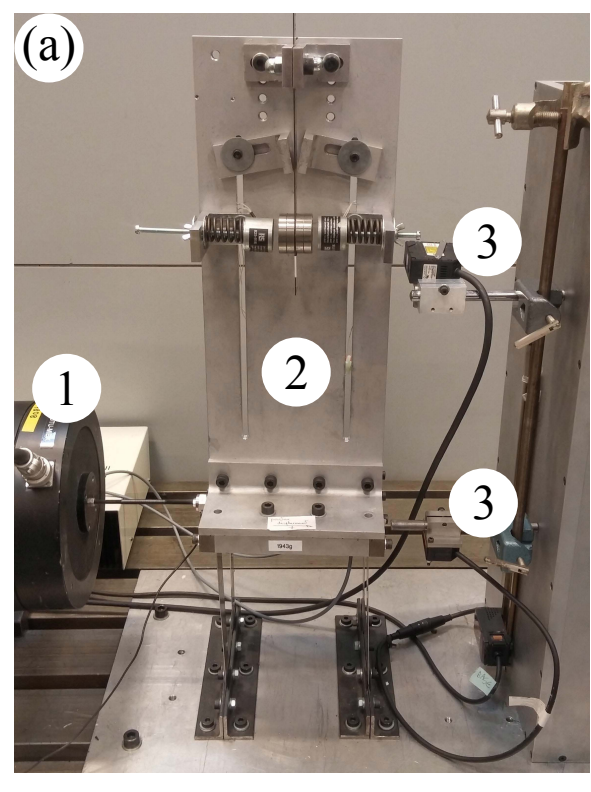

(b)
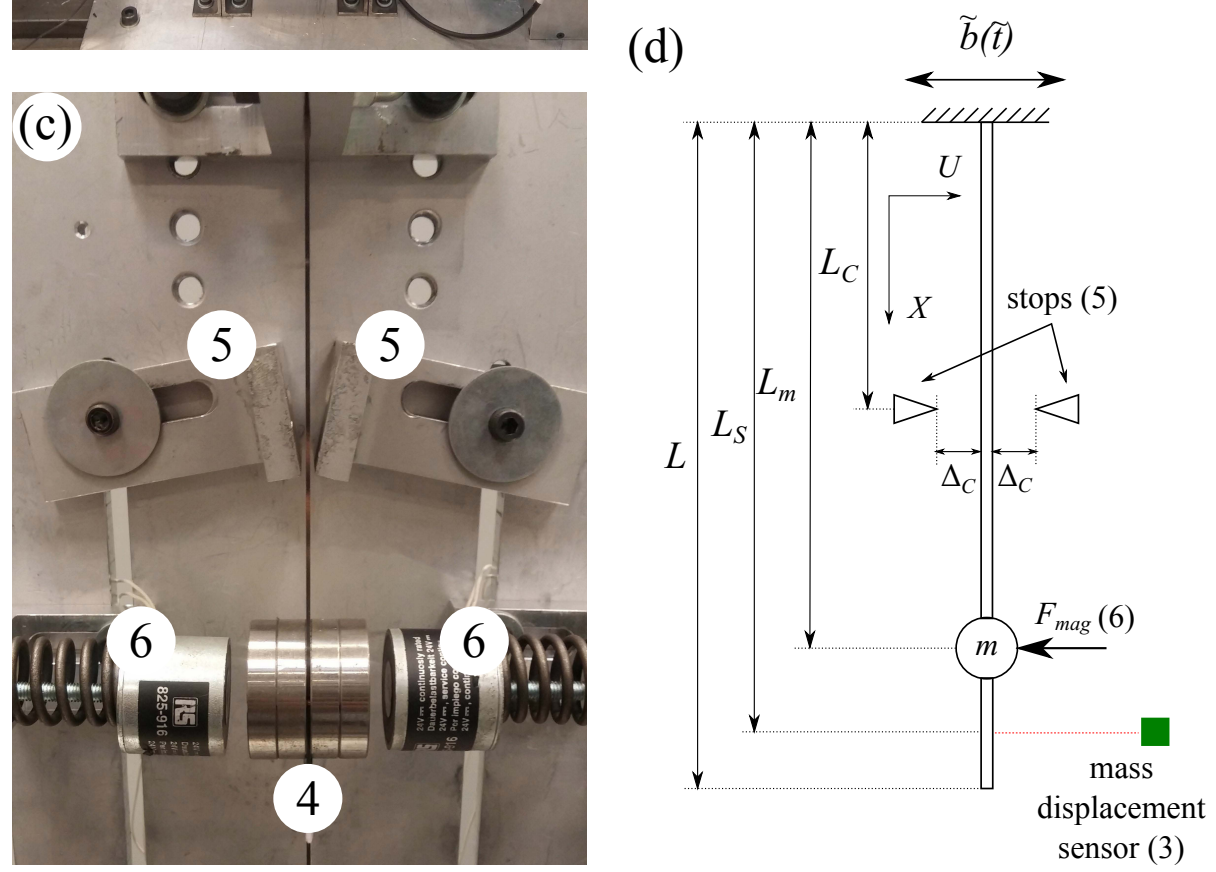

Figure 1: Experimental setup $(\mathrm{a}, \mathrm{c})$ and its schematic representation $(\mathrm{b}, \mathrm{d})$. 

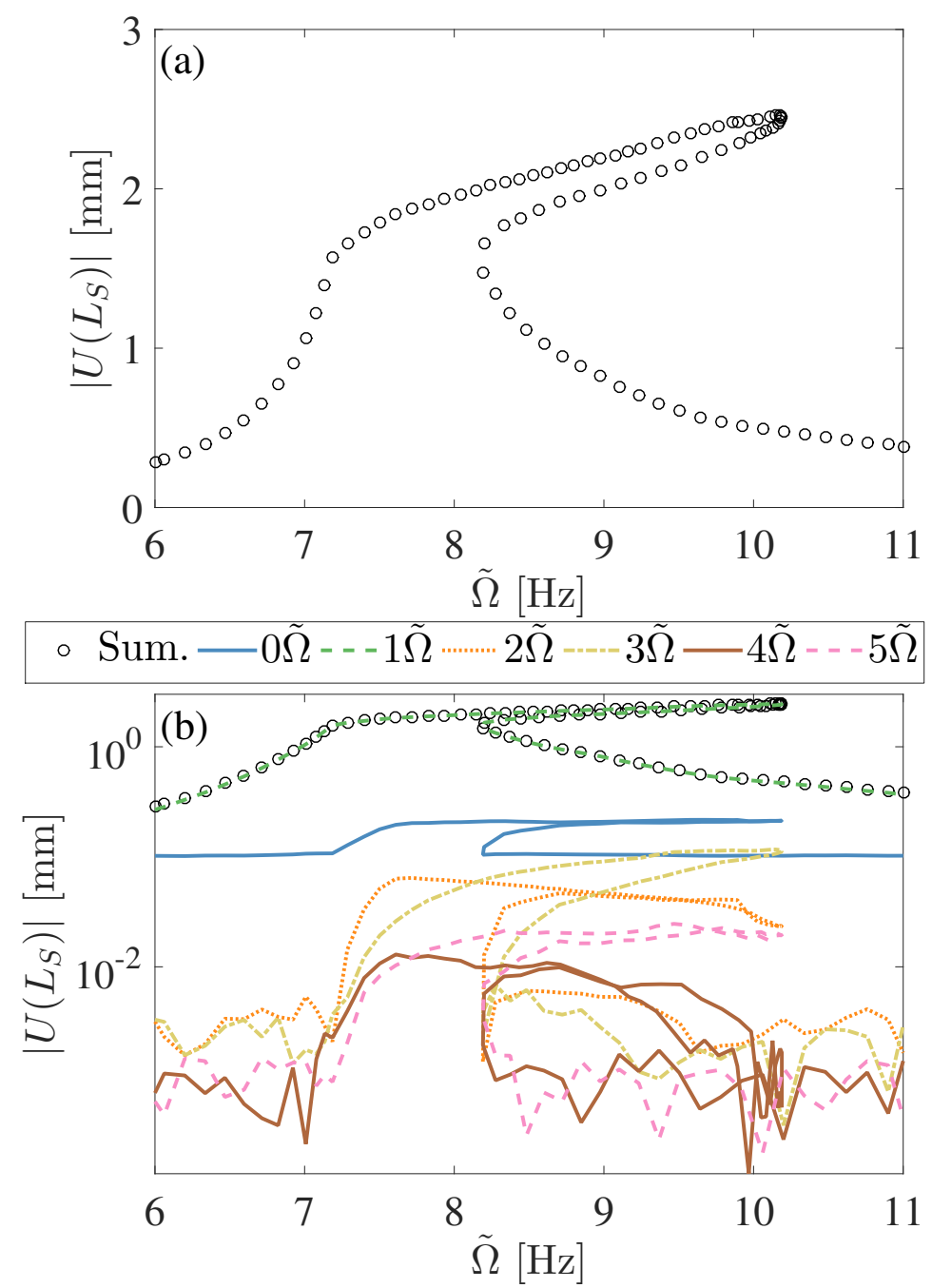

Figure 2: (a) Experimental frequency response and its (b) harmonic decomposition 


\section{Equations of motion}

As the measurement and impact locations $L_{S}$ and $L_{C}$ are not coincident and due to beam deflection, the measured grazing amplitude $\Delta_{G}$ will differ from the gap width $\Delta_{C}$. This difference can be seen in Fig. 3 for a cantilever beam oscillating at its first mode. The deflection is caused by the inertial force $F=-m \ddot{U}\left(L_{m}, \tilde{t}\right)$. The measured grazing amplitude will be used to normalize the equations of motion later on and to define impact force models in Section 4

The electrodynamic shaker is not feedback controlled, being not able to provide constant excitation amplitude on the investigated frequency range. Also, the displacement of the sub-system shaker-platform around resonance is affected by a nonlinear coupling with the impacting beam as mentioned in [30]. Alternatively, one could consider the single harmonic electric signal fed into the shaker's power amplifier as the system's external excitation, since it has constant amplitude (in volts). This electric signal excites the shaker-platform subsystem, which in turn excites the impacting beam.

So, the base excitation can be written as:

$$
\tilde{b}(\tilde{t})=\tilde{\gamma} \tilde{V} \sin (\tilde{\Omega} \tilde{t})
$$

where $\tilde{V}$ is the electric amplitude in $\mathrm{V}$ and $\tilde{\gamma}$ is the unit conversion gain in $\mathrm{m} / \mathrm{V}$. In the schematic representation of the forced impacting beam in Fig. 11d, $L_{C}, L_{m}, L_{S}$ and $L$ represent the axial location of the stops, lumped mass, displacement sensor and beam length, respectively, $\Delta_{C}$ represents the gap width. Using Bernoulli-Euler beam theory, one can write its equation of motion for transverse displacement $U(X, \tilde{t})$ as [14]:

$$
\begin{array}{r}
\left(\rho A+m \delta\left(X-L_{m}\right)\right) \ddot{U}+D E I \dot{U}^{\prime \prime \prime \prime}+E I U^{\prime \prime \prime \prime}+\tilde{f}_{C}(U, \dot{U}) \delta\left(X-L_{C}\right)= \\
\left(\rho A+m \delta\left(X-L_{m}\right)\right) \tilde{\Omega}^{2} \gamma \tilde{V} \sin (\tilde{\Omega} \tilde{t}), \\
U(0, \tilde{t})=U^{\prime}(0, \tilde{t})=U^{\prime \prime}(L, \tilde{t})=U^{\prime \prime \prime}(L, \tilde{t})=0,
\end{array}
$$

where $(\cdot) \equiv \partial(\cdot) / \partial \tilde{t},(\cdot)^{\prime} \equiv \partial(\cdot) / \partial X, \rho, E, A$ and $I$ represents the beam's density, elasticity modulus, cross-sectional area and cross-section area moment of inertia, respectively, $m$ is the lumped mass and $\delta(\cdot)$ is Dirac's delta function. The function $\tilde{f}_{C}(U, \dot{U})$ represents the transverse impact force, resulting from the contact between beam and 


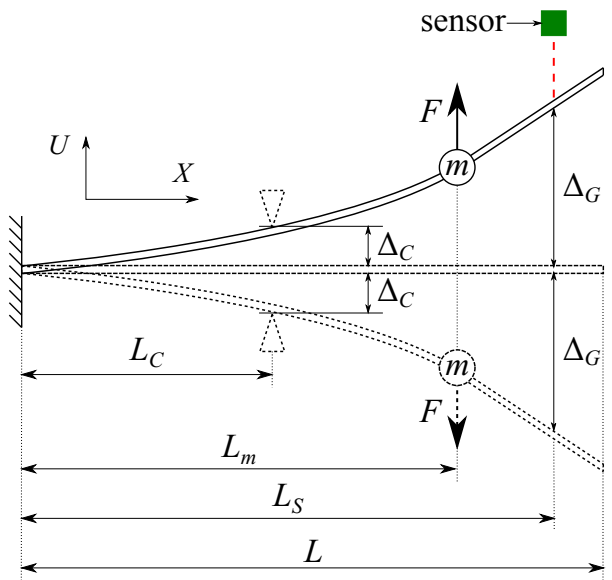

Figure 3: Deflection of a cantilever beam.

stops. This function can be modeled by several means from which three will be addressed in Section 4

The present model is similar to the one proposed by Elmegård et al. [14], which did not define an impact force explicitly but used a piecewise relation for the spatial discretization of the equations of motion.

One way of inserting the stiffness proportional damping coefficient $D$ in the BernoulliEuler model is to assume the beam's material to have a viscoelastic behavior, described by Kelvin and Voigt's (KV) model. Assuming that lumped mass is much bigger than the beam's mass, i.e. $m \gg \rho A L$, and defining $U=u \Delta_{G}$ and $X=x L$ as normalized transverse displacement and axial position, one arrives at:

$$
\begin{array}{r}
\delta\left(x-x_{m}\right) \ddot{u}+\left(D \dot{u}^{\prime \prime \prime \prime}+u^{\prime \prime \prime \prime}\right) E I /\left(m L^{3}\right)+ \\
+\tilde{f}_{C}\left(u \Delta_{G}, \dot{u} \Delta_{G}\right) \delta\left(x-x_{C}\right) /\left(m \Delta_{G}\right)=\delta\left(x-x_{m}\right) \tilde{V}\left(\tilde{\gamma} / \Delta_{G}\right) \tilde{\Omega}^{2} \sin (\tilde{\Omega} \tilde{t}),
\end{array}
$$

where now $(\cdot)^{\prime} \equiv \partial(\cdot) / \partial x, x_{C}=L_{C} / L$, and $x_{m}=L_{m} / L$. This system can be spatially discretized by defining:

$$
u(x, \tilde{t})=\sum_{i=1}^{N} \phi_{i}(x) q_{i}(\tilde{t}),
$$

with the mode shapes $\phi_{i}(x)$ satisfying all essential boundary conditions and $q_{i}(\tilde{t})$ as the modal coordinates. When the discretized version of Eq. (4) is driven around its first 
resonance the high order modes $(i \geq 2)$ are expected to have low influence because they are increasingly damped by the stiffness proportional damping. The weak significance of higher modes for the experimental setup was already illustrated by Fig. 2p. So, a single-DOF discretization of Eq. (4) appears to be an appropriate model for the experimental setup around its first resonance. Using standard Galerkin approximation this model is obtained as:

$$
\ddot{q}+(D \dot{q}+q) \omega_{n}^{2}+\bar{f}_{C}(q, \dot{q})=\tilde{\Omega}^{2} \gamma \tilde{V} \sin (\tilde{\Omega} \tilde{t}),
$$

where:

$$
\begin{array}{r}
\omega_{n}^{2}=K_{S} / M_{S}, \quad M_{S}=m \phi\left(x_{m}\right)^{2}, \\
K_{S}=\frac{E I}{L^{3}} \int_{0}^{1}\left(\phi^{\prime \prime}(x)\right)^{2} \mathrm{~d} x, \quad \gamma=\frac{\tilde{\gamma}}{\phi\left(x_{m}\right) \Delta_{G}}, \\
\bar{f}_{C}(q, \dot{q})=\frac{\phi\left(x_{C}\right)}{M_{S} \Delta_{G}} \tilde{f}_{C}\left(\phi\left(x_{C}\right) q \Delta_{G}, \phi\left(x_{C}\right) \dot{q} \Delta_{G}\right),
\end{array}
$$

are the squared fundamental linear natural frequency, equivalent structural mass and stiffness coefficient, and normalized unit conversion gain and impact force, respectively. Additionally, $\phi(\cdot)$ is the static deformation pattern of a cantilever beam loaded at $x=x_{m}$. Defining the structural damping ratio as $\beta_{S}=\frac{1}{2} D \omega_{n}$, substituting it into Eq. (4) and normalizing time $\tilde{t}$ using the system's fundamental linear natural frequency $\omega_{n}$, one arrives at:

$$
\ddot{q}+2 \beta_{S} \dot{q}+q+f_{C}(q, \dot{q})=\Omega^{2} \gamma \tilde{V} \sin (\Omega t)
$$

where:

$$
t=\omega_{n} \tilde{t}, \quad \Omega=\tilde{\Omega} / \omega_{n} \quad \text { and } \quad f_{C}(q, \dot{q})=\bar{f}_{C}\left(q, \omega_{n} \dot{q}\right) / \omega_{n}^{2}
$$

are the normalized time, normalized forcing frequency, and nondimensional impact force, respectively. Also, from now on $(\cdot) \equiv \partial(\cdot) / \partial t$.

The vibro-impact oscillations measured from the experimental setup can be related to Eq. 10 by multiplying the model solution by the measured grazing amplitude and by the first mode shape at the measurement location, $U\left(L_{S}, \tilde{t}\right)=q\left(\omega_{n} \tilde{t}\right) \Delta_{G} \phi\left(L_{S} / L\right)$. 
This relation can be even simpler if the first mode shape is normalized with respect to the measurement location, $L_{S}$. By doing so, the measured vibrations become proportional to the grazing amplitude, i.e., $U\left(L_{S}, \tilde{t}\right)=q\left(\omega_{n} \tilde{t}\right) \Delta_{G}$.

\section{Impact force models}

As mentioned in Section 1, there are several ways of modeling vibro-impact forces. In this work three impact force models will be considered. The piecewise-linear KelvinVoigt model, the Power-law function impact force and a combination of the two called the modified Kelvin-Voigt model. This section describes these models, showing their physical basis, advantages and limitations.

\subsection{Kelvin-Voigt model}

The mass and stops are mounted in different locations of the beam, being not coincident $\left(L_{m} \neq L_{C}\right)$, see Fig. 1 , d. Thus the vibrations of the lumped mass are not directly constrained by the stops, allowing points of the beam to suffer further deformations beyond those necessary to reach the stops. With first-mode beam oscillations, in a contact configuration only a segment of the beam, from $L_{C}$ to $L_{m}$, suffers further deformation. This segment can be considered as a lumped mass beam in a pinned-free configuration, with its own dynamic properties, such as natural frequency and damping.

So one can model the contact configuration applying Kelvin-Voigt's approach to the beam segment as well. This secondary system is inactive by default, being active only if the measured transverse mass displacement exceeds the measured grazing amplitude, $\Delta_{G}$. For symmetric bilateral impacts:

$$
\tilde{f}_{C}(U, \dot{U})=\left\{\begin{array}{cl}
0, & \text { if }\left|U_{C}\right| \leq \Delta_{C}, \\
\left(K_{C}-K_{S}\right)\left(U_{C}-\Delta_{C}\right)+D_{C} \dot{U}_{C}, & \text { if } U_{C} \geq \Delta_{C} \\
\left(K_{C}-K_{S}\right)\left(U_{C}+\Delta_{C}\right)+D_{C} \dot{U}_{C}, & \text { if } U_{C} \leq-\Delta_{C}
\end{array}\right.
$$

where $K_{C}$ and $D_{C}$ are the stiffness and damping coefficients for the beam in the contact configuration and $U_{C}=U\left(L_{C}, \tilde{t}\right)$ because the force it is being applied at $L_{C}$. Its nondimensional version can be obtained by applying the same steps taken from Eq. (2) to Eq. 10, giving: 


$$
f_{C}(q, \dot{q})=\left\{\begin{array}{cl}
0, & \text { if }|q| \leq 1, \\
\phi_{C}^{2}\left(\left(\omega_{R}^{2}-1\right)(q-1)+2 \beta_{C} \omega_{R} \dot{q}\right), & \text { if } q \geq 1, \\
\phi_{C}^{2}\left(\left(\omega_{R}^{2}-1\right)(q+1)+2 \beta_{C} \omega_{R} \dot{q}\right), & \text { if } q \leq-1,
\end{array}\right.
$$

where:

$$
\beta_{C}=\frac{D_{C}}{2 M_{S} \omega_{C}}, \quad \omega_{C}^{2}=K_{C} / M_{S}, \quad \omega_{R}=\omega_{C} / \omega_{n}, \quad \phi_{C}=\phi\left(x_{C}\right)=\frac{\Delta_{C}}{\Delta_{G}}
$$

are the contact damping ratio, the contact natural frequency, the natural frequency ratio and the cantilever beam mode shape evaluated at $x_{C}$, which is equal to $\Delta_{C} / \Delta_{G}$.

The factor $\phi_{C}$ is purely geometric, accounting for the distance between impact and measurement points. For the experimental setup in Fig. 1 . $\phi_{C}=0.241$. Comparing the locations of measurement and impact on the beam, see Figs. 1 and 3 , one can see that the contact point is closer to the beam's clamped end than the measurement point, so for first mode oscillations, $\phi_{C}<1$ attenuating the impact force. On the other hand, swapping impact and measurement positions would strengthen the impact force.

Despite its straightforward physical interpretation, Fig. 4a illustrates that KV's model dissipative term is discontinuous with respect to velocity. Also, the impact force changes its sign at the end of the contact, indicating that the impacting element is being pulled towards the stop again, a situation which does not make sense from the physical point of view.

Besides the drawbacks mentioned previously, the impact condition enables one to estimate the impact duration. From the contact intervals (where $|q| \geq 1$ ) shown in Fig. 4 p, one can define the normalized contact duration for a full oscillation as:

$$
\tau=2\left(\pi-2 \varphi_{0}\right) / \Omega
$$

where $\varphi_{0}=\arcsin (1 / Q)$ is the grazing angle and $Q$ is the first-order modal amplitude, which heavily depends on the model parameters, $\omega_{R}$ and $\beta_{C}$. When using this model, one can estimate the normalized contact duration for any pair $(\Omega, Q)$ as shown in Fig. 怍, where curves of constant $\tau$ are presented with normalized experimental data (crosses). The experimental data was taken from Fig. 17 and normalized using the fundamental linear natural frequency and the measured grazing amplitude, given by $f_{n} \approx$ 

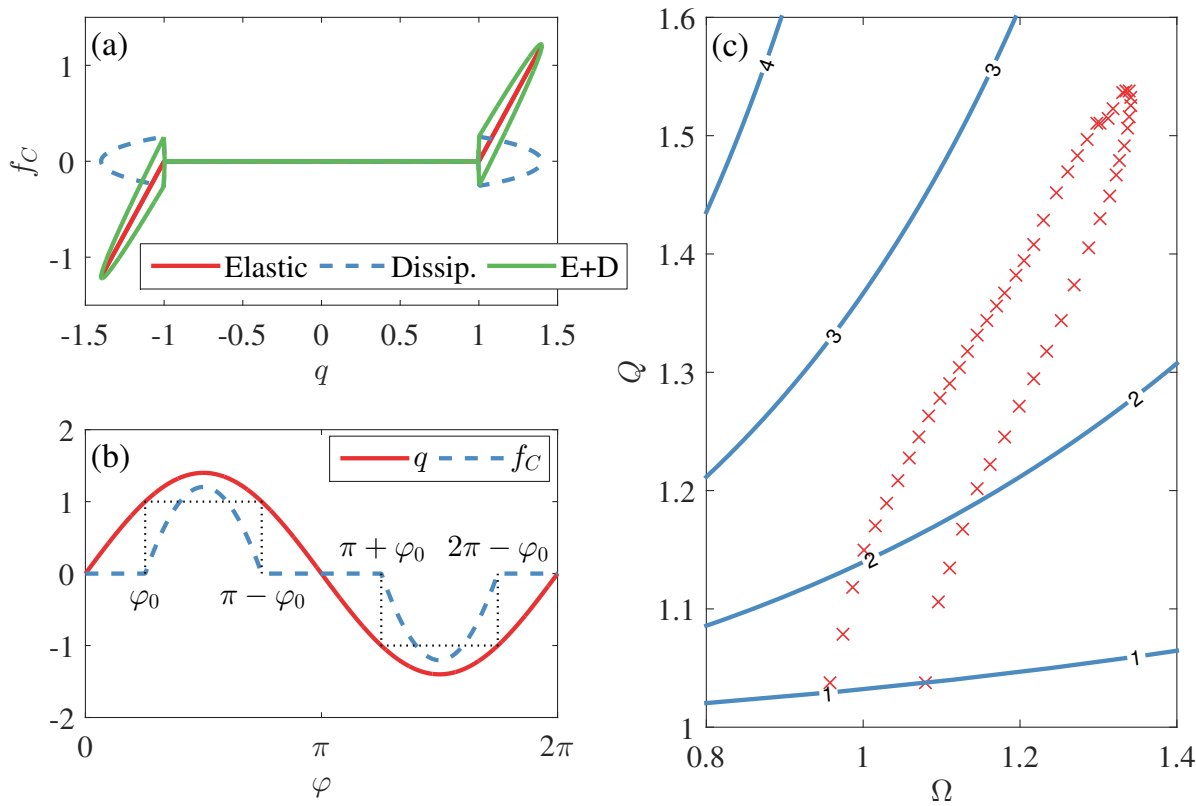

Figure 4: (a): Elastic and dissipative components of the piecewise linear impact force model $\left(\omega_{R}=\right.$ 7.278, $\beta_{C}=0.258$ and $\phi_{C}=0.241$ ). (b): Time series of elastic Kelvin-Voigt impact force together with $q=Q \sin (\varphi)$. (c): Normalized experimental data (red x's) and curves of constant normalized contact duration (blue lines), the numbers on each curve represent the contact duration. 
$7.6 \mathrm{~Hz}$ and $\Delta_{G} \approx 1.6 \mathrm{~mm}$, respectively. As expected, the contact duration grows for higher amplitudes $Q$.

\subsection{Power-law impact force model}

The drawbacks of Kelvin and Voigt's model can be overcome by approximating the impact force by a power function. However, instead of considering powers of impact deformation $\left(U_{C} \pm \Delta_{C}\right)$ as done by Hunt and Crossley [4], one can consider powers of the ratio between displacement and the gap width, $U_{C} / \Delta_{C}$. With this approach, one can define a smooth force impact which is weak in the region $\left|U_{C}\right|<\Delta_{C}$ but grows very fast around the gap width, $\pm \Delta_{C}$. Also, the participation of the Power-law impact force on the non-contact regime is not necessarily a problem; a similar effect can actually occur due to a small measurement error on the gap width, shifting the ratio $U_{C} / \Delta_{C}$ a little bit away from 1, which is the value where the power function starts to grow faster. Also, the participation in the non-contact phase can be increased/decreased according to model parameters. For symmetric bilateral impacts, the elastic force should be an odd exponent power, while the dissipative force should have an even exponent term multiplying the velocity. So:

$$
\tilde{f}_{C}(U, \dot{U})=F\left(\frac{U_{C}}{\Delta_{C}}\right)^{2 n-1}+D_{C}\left(\frac{U_{C}}{\Delta_{C}}\right)^{2 p} \dot{U}_{C}
$$

where $n \geq 1$ and $p \geq 0$ are integer exponents for the restoring and dissipative terms, respectively. As $U_{S} / \Delta_{G}$ is non dimensional, $D_{C}$ has the same physical dimension of a linear viscous damping coefficient, while $F$ has dimensions of force. So, considering $F=K_{C} \Delta_{G}$ and applying the same steps as from Eq. (2) to Eq. (10), one can obtain the non-dimensional Power-law impact force as:

$$
f_{C}(q, \dot{q})=\phi_{C} \omega_{R}^{2} q^{2 n-1}+2 \beta_{C} \omega_{R} q^{2 p} \dot{q} \phi_{C}^{2},
$$

where $\beta_{C}, \phi_{C}$ and $\omega_{R}$ were defined previously for the $\mathrm{KV}$ force model. However, it should be mentioned that despite having dynamic parameters with the same name and definition, the models are not equivalent. With the exception of $\phi_{C}$, one should not expect their parameters to have similar numeric values or physical meaning. For instance, 


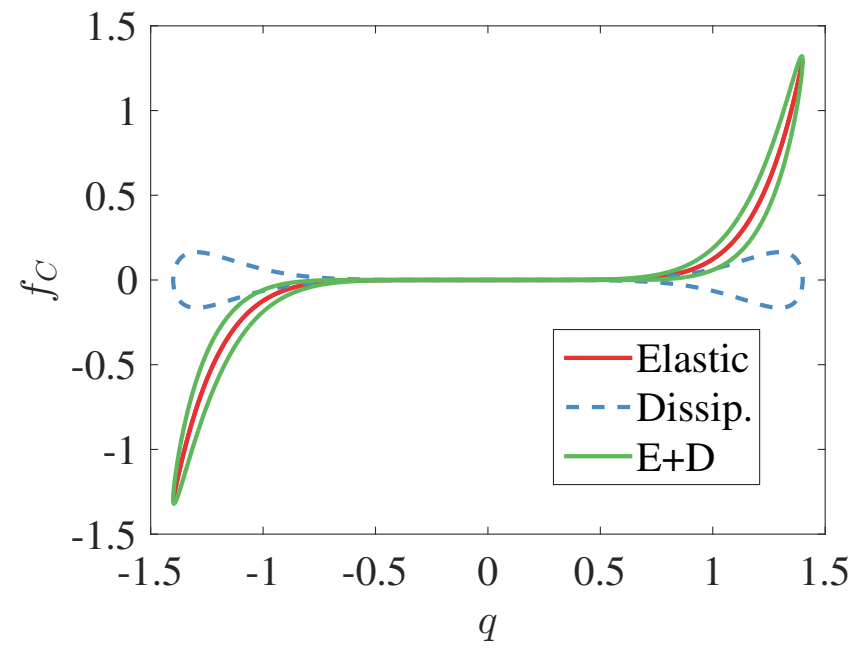

Figure 5: Elastic and dissipative components of Power-law impact force model for $q=Q \sin (\varphi), \omega_{R}=$ $0.715, \beta_{C}=0.659, n=4, p=3$ and $\phi_{C}=0.241$.

the terms $\omega_{R}$ and $\beta_{C}$ have no physical meaning for the Power-law model. Also, notice that $\phi_{C}$ affects the restoring and dissipative parts of the impact force differently.

Comparing Fig. 4a and Fig. 5, the smoothness of the Power-law model is clearly seen. Instead of jumping to a nonzero value, the dissipative Power-law force grows smoothly. However, nonphysical sign changes similar to KV model are possible if the model is purely dissipative, $\omega_{R} \equiv 0$.

Finally, it is important to remember that numeric simulations involving the Powerlaw model become demanding for high $n$ and $p$, due to the stiffening of the ODE, losing the advantage of being easier to simulate than the $\mathrm{KV}$ model, which is given by a piecewise linear function.

\subsection{Modified Kelvin-Voigt model}

While the Power-law model solves the problems presented by Kelvin and Voigt's model, it also removes most of the physical meaning of the parameters involved in the impact phenomenon. In order to obtain the expected behavior for the impact force while keeping part of the physical insight given by the KV approach, a combination of both models is presented. 
As the dissipative term seems to be the problematic part of the $\mathrm{KV}$ model, one can multiply it by a power of the displacement. However, instead of using $U_{C} / \Delta_{C}$ as power term, one can add \pm 1 to it, obtaining $\left(U_{C} / \Delta_{C} \pm 1\right)$. In this way, it is assured that the damping term starts to grow smoothly from zero, without any discontinuity. Also, this term can be rewritten as $\left(U_{C} \pm \Delta_{C}\right) / \Delta_{C}$ which can be interpreted as a mechanical impact strain. Another option would be using the impact deformation $\left(U_{C} \pm \Delta_{C}\right)$ as power term, but this term is highly dependent on the gap width, which is very small and would demand an extremely high damping coefficient to compensate for it. So, the combined model is given by:

$$
\tilde{f}_{C}(U, \dot{U})=\left\{\begin{array}{cl}
0, & \text { if }\left|U_{C}\right| \leq \Delta_{C}, \\
\left(K_{C}-K_{S}\right)\left(U_{C}-\Delta_{C}\right)+D_{C} \dot{U}_{C}\left(\frac{U_{C}}{\Delta_{C}}-1\right)^{2 p}, & \text { if } U_{C} \geq \Delta_{C} \\
\left(K_{C}-K_{S}\right)\left(U_{C}+\Delta_{C}\right)+D_{C} \dot{U}_{C}\left(\frac{U_{C}}{\Delta_{C}}+1\right)^{2 p}, & \text { if } U_{C} \leq-\Delta_{C}
\end{array}\right.
$$

which leads to the original $\mathrm{KV}$ model if $p=0$. The nondimensionalization of this expression gives:

$$
f_{C}(q, \dot{q})=\left\{\begin{array}{cl}
0, & \text { if }|q| \leq 1, \\
\phi_{C}^{2}\left(\left(\omega_{R}^{2}-1\right)(q-1)+2 \beta_{C} \omega_{R} \dot{q}(q-1)^{2 p}\right), & \text { if } q \geq 1, \\
\phi_{C}^{2}\left(\left(\omega_{R}^{2}-1\right)(q+1)+2 \beta_{C} \omega_{R} \dot{q}(q+1)^{2 p}\right), & \text { if } q \leq-1,
\end{array}\right.
$$

with $\beta_{C}$ and $\omega_{R}$ being the same as before and $\omega_{R}$ keeping its straightforward physical meaning. This model can be seen as a particular case of Hunt and Crossley's model [4], which originally assumed the elastic term to be a power function as well.

The advantages of mixing both models can be seen by comparing Fig. 6 with Fig. 4 a and Fig. 5. While the original KV model changes its sign during impact, leading to nonphysical situations, and the Power-law model is active also in the non-contact phase, the combined model has none of this undesired behaviors. Also, it is possible to obtain the normalized contact duration for a full oscillation using this model, shown in Fig. 4p and given by Eq. 15. However, all the three models considered in this work present nonphysical sign changes, when elastic forces are neglected. 


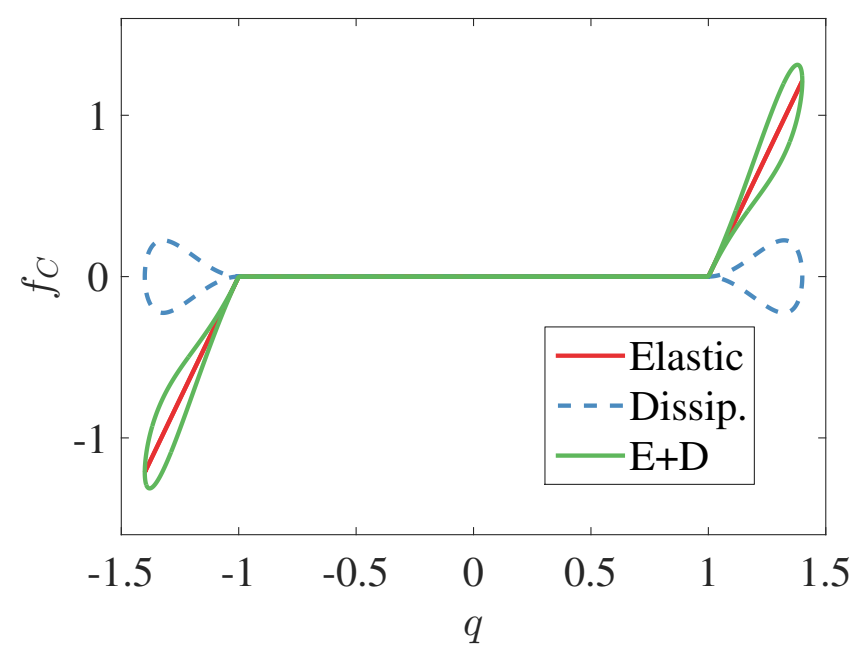

Figure 6: Elastic and dissipative components of modified Kelvin-Voigt impact force model for $q=Q \sin (\varphi)$, $\omega_{R}=7.306, \beta_{C}=4.636, p=1$ and $\phi_{C}=0.241$.

where:

$$
a_{1}=\frac{2}{T} \int_{0}^{T} f_{C}(q(t), \dot{q}(t)) \sin (\Omega t) \mathrm{d} t, \quad b_{1}=\frac{2}{T} \int_{0}^{T} f_{C}(q(t), \dot{q}(t)) \cos (\Omega t) \mathrm{d} t
$$


are Fourier coefficients, $\Omega$ is the forcing frequency and $T$ is the forcing period. To obtain some physical insight, this approximation can be rearranged as a spring-dashpot system, $f_{C}(q, \dot{q}) \approx \kappa q+2 \sigma \dot{q}$, whose coefficients can be found by comparing the responses of its mechanical and Fourier approximations to a solution $q(t)=Q \sin (\Omega t+\theta)$, as follows:

$$
f_{C}(q(t), \dot{q}(t)) \approx \kappa Q \sin (\Omega t+\theta)+2 \sigma \Omega Q \cos (\Omega t+\theta) \approx a_{1} \sin (\Omega t)+b_{1} \cos (\Omega t)
$$

Defining $\varphi=\Omega t+\theta$, the harmonic linearization coefficients $\kappa$ and $\sigma$ can be defined as:

$$
\begin{gathered}
\kappa=\frac{1}{\pi Q} \int_{0}^{2 \pi} f_{C}(q(\varphi), \dot{q}(\varphi)) \sin \varphi \mathrm{d} \varphi, \\
\sigma=\frac{1}{2 \pi Q \Omega} \int_{0}^{2 \pi} f_{C}(q(\varphi), \dot{q}(\varphi)) \cos \varphi \mathrm{d} \varphi .
\end{gathered}
$$

Substituting the spring-dashpot approximation into Eq. (10) leads to:

$$
\ddot{q}+2\left(\beta_{S}+\sigma\right) \dot{q}+(1+\kappa) q=\Omega^{2} \gamma \tilde{V} \sin (\Omega t),
$$

which corresponds to a standard mechanical oscillator with a pair of springs and dampers in parallel. Its stationary frequency response is given by $q(t)=Q \sin (\Omega t+\theta)$, where:

$$
Q=\frac{\Omega^{2} \gamma \tilde{V}}{\sqrt{\left(\Omega^{2}-1-\kappa\right)^{2}+4 \Omega^{2}\left(\beta_{S}+\sigma\right)^{2}}},
$$

or:

$$
Q^{2}\left(\left(\Omega^{2}-1-\kappa\right)^{2}+4 \Omega^{2}\left(\beta_{S}+\sigma\right)^{2}\right)=\left(\Omega^{2} \gamma \tilde{V}\right)^{2} .
$$

The difference between the current case and a standard mechanical oscillator is that the harmonic linearization coefficients $\kappa$ and $\sigma$ depend of the response amplitude, $Q$. Both coefficients will be defined in Section 5.3 for different models of the impact force. 


\subsection{Averaging}

Standard first-order averaging [34] implicitly uses the same assumption made for harmonic linearization, that higher harmonics can be neglected and the solution can be approximated by a single harmonic expression, $q(t) \approx Q \sin \varphi, \varphi=\Omega t+\theta$. However standard averaging is restricted to weakly nonlinear systems only [35], i.e., systems with small forcing amplitude $\tilde{V}$, damping $\beta_{S}$ and nonlinearities $f_{C}(q, \dot{q})$. The equation of motion can be written as a weakly nonlinear system in the following way:

$$
\ddot{q}+q=\epsilon\left(\Omega^{2} \gamma \tilde{V} \sin (\Omega t)-2 \beta_{S} \dot{q}-f_{C}(q, \dot{q})\right)
$$

where the parameter $\epsilon \ll 1$ indicates which terms are small in comparison with linear terms. Another feature of the standard averaging is that it allows both amplitude and phase to vary in time, i.e. $Q=Q(t)$ and $\theta=\theta(t)$. Defining the time-derivative of the solution as $\dot{q}(t) \approx \Omega Q \cos \varphi$ implies that $\dot{\theta} Q \cos \varphi=-\dot{Q} \sin \varphi$. Substituting these relations into Eq. (28) results in a system of differential equations in terms of amplitude and phase:

$$
\begin{aligned}
\Omega \dot{Q} & =\frac{1}{2} Q\left(\Omega^{2}-1\right) \sin 2 \varphi+\epsilon\left(\operatorname { c o s } \varphi \left(\gamma \tilde{V} \Omega^{2} \sin (\varphi-\theta)+\right.\right. \\
& \left.\left.-f_{C}(q, \dot{q})\right)-2 \Omega \beta_{S} Q \cos ^{2} \varphi\right) \\
\Omega Q \dot{\theta} & =-Q\left(\Omega^{2}-1\right) \sin ^{2} \varphi+\epsilon\left(\operatorname { s i n } \varphi \left(f_{C}(q, \dot{q})+\right.\right. \\
& \left.\left.-\gamma \tilde{V} \Omega^{2} \sin (\varphi-\theta)\right)+\beta_{S} \Omega Q \sin 2 \varphi\right)
\end{aligned}
$$

Almost all terms on the right-hand sides of these equations are multiplying $\epsilon$ and therefore are small. Around the primary external resonance the term $\Omega^{2}-1$ is also small and so the whole right-hand side of these differential equations is small, which means that both $Q$ and $\theta$ vary slowly with time. In this case one can approximate Eqs. 29) and (30) by their average over one period of oscillations, obtaining:

$$
\begin{gathered}
2 \pi \Omega \dot{Q}=-\epsilon\left(2 \pi \Omega \beta_{S} Q+\pi \gamma \tilde{V} \Omega^{2} \sin \theta+\int_{0}^{2 \pi} f_{C}(q(\varphi), \dot{q}(\varphi)) \cos \varphi \mathrm{d} \varphi\right), \\
2 \pi \Omega Q \dot{\theta}=-\epsilon \pi Q\left(\Omega^{2}-1\right)+\epsilon\left(\int_{0}^{2 \pi} f_{C}(q(\varphi), \dot{q}(\varphi)) \sin \varphi \mathrm{d} \varphi-\pi \gamma \tilde{V} \Omega^{2} \cos \theta\right),
\end{gathered}
$$


where $\epsilon$ is multiplying the term $\left(\Omega^{2}-1\right)$ to explicitly indicate that this term is small. The integrals of the impact force can be replaced by the harmonic linearization coefficients $\kappa, \sigma$ given by Eqs. (23) and (24). The equilibria of the resulting system correspond to the steady-state response of Eq. [10, so:

$$
\begin{aligned}
\Omega^{2} \gamma \tilde{V} \sin \theta+2 \Omega Q\left(\beta_{S}+\sigma\right) & =0, \\
Q\left(1+\kappa-\Omega^{2}\right)-\Omega^{2} \gamma \tilde{V} \cos \theta & =0 .
\end{aligned}
$$

Eliminating $\theta$ from the last expressions gives:

$$
\left(\gamma \tilde{V} \Omega^{2}\right)^{2}=Q^{2}\left(\left(\Omega^{2}-1-\kappa\right)^{2}+4 \Omega^{2}\left(\beta_{S}+\sigma\right)^{2}\right),
$$

which is identical to Eq. 27]. This same relation could be obtained using Multiple Scales or modified Lindstedt-Poincaré [24] methods, but Averaging was chosen due to its well developed mathematical basis and effectiveness for other vibro-impacting systems [21, 22]. In this context, despite its loose mathematical basis, Harmonic Linearization can be seen as a shortcut method, giving the same results for stationary solutions as Averaging, but bypassing its intermediate steps such as defining small terms, obtaining a system of differential equations for amplitude and phase and finding its equilibrium state.

\subsection{Application to specific impact force models}

\subsubsection{Kelvin-Voigt impact force}

As mentioned previously, Kelvin-Voigt's impact force is not active during the whole period of oscillation. So, the limits of integration in Eqs. (23) and (24) can be restricted to the contact period, i.e. for $\varphi \in\left[\varphi_{0} ; \pi-\varphi_{0}\right]$ for $q \geq 1$ and $\varphi \in\left[\pi+\varphi_{0} ; 2 \pi-\varphi_{0}\right]$ for $q \leq-1$, as shown in Fig. 4b. Considering $q \approx Q \sin \varphi$ and substituting Eq. (13) into Eqs. (23) and (24) leads to:

$$
\begin{aligned}
\kappa & =\phi_{C}^{2}\left(\omega_{R}^{2}-1\right) W(Q), \\
\sigma & =\phi_{C}^{2} \beta_{C} \omega_{R} W(Q),
\end{aligned}
$$

where: 


$$
W(Q)=1-\frac{2}{\pi}\left(\varphi_{0}+\frac{1}{2} \sin \left(2 \varphi_{0}\right)\right), \quad \varphi_{0}=\arcsin (1 / Q),
$$

is a function of the forcing amplitude $Q$. Despite its complicated formula, $W(Q)$ does not grow unbounded, being limited between 0 and 1 for $Q$ between 1 and infinity. Substituting $\kappa$ and $\sigma$ in the frequency response, Eq. (27) leads to:

$$
Q^{2}\left(\left(\Omega^{2}-1-\phi_{C}^{2}\left(\omega_{R}^{2}-1\right) W(Q)\right)^{2}+4 \Omega^{2}\left(\beta_{S}+\phi_{C}^{2} \beta_{C} \omega_{R} W(Q)\right)^{2}\right)-\left(\Omega^{2} \gamma \tilde{V}\right)^{2}=0
$$

As $W(Q)$ varies between 0 and 1, it works as a tuning parameter between two linear oscillators, one given by Eq. 10 and $f_{C} \equiv 0$, and another given by the same equation and $f_{C} \equiv 0$, but with natural frequency $\omega_{R}$ instead of unity and damping $\beta_{S}+\beta_{C} \omega_{R}$ instead of $\beta_{S}$. The frequency response equations for these oscillators are given by respectively:

$$
\begin{array}{r}
Q^{2}\left(\left(\Omega^{2}-1\right)^{2}+4 \Omega^{2} \beta_{S}^{2}\right)-\left(\Omega^{2} \gamma \tilde{V}\right)^{2}=0, \\
Q^{2}\left(\left(\Omega^{2}-\phi_{C}^{2} \omega_{R}^{2}\right)^{2}+4 \Omega^{2}\left(\beta_{S}+\phi_{C}^{2} \beta_{C} \omega_{R}\right)^{2}\right)-\left(\Omega^{2} \gamma \tilde{V}\right)^{2}=0 .
\end{array}
$$

\subsubsection{Power-law impact force}

Substituting Eq. (17) into Eqs. (23) and (24) leads to:

$$
\begin{aligned}
\kappa & =2 \phi_{C} G_{n} \frac{\omega_{R}^{2}}{Q^{2}}, \\
\sigma & =\phi_{C}^{2} \beta_{C} \omega_{R} \frac{G_{p}}{p+1},
\end{aligned}
$$

where:

$$
G_{j}=\frac{Q^{2 j}}{\sqrt{\pi}} \frac{\Gamma(j+1 / 2)}{\Gamma(j+1)}, \quad j=n, p
$$

is a function of the exponent $n$ or $p$ and the steady-state amplitude $Q$ and $\Gamma$ denotes the Gamma function. As expected for a power-function term, $G_{j}$ grows unbounded for $j \rightarrow \infty$ and $|Q|>1$. Substituting $\kappa$ and $\sigma$ in the frequency response equation, Eq. (27) gives:

$$
Q^{2}\left(\left(\Omega^{2}-1-2 \phi_{C} G_{n} \omega_{R}^{2} / Q^{2}\right)^{2}+4 \Omega^{2}\left(\beta_{S}+\phi_{C}^{2} \beta_{C} \omega_{R} G_{p} /(p+1)\right)^{2}\right)-\left(\Omega^{2} \gamma \tilde{V}\right)^{2}=0
$$




\subsubsection{Modified Kelvin-Voigt impact model}

The equivalent stiffness $\kappa$ is given by Eq. (36) and the equivalent damping is given by:

$$
\begin{aligned}
\pi \sigma & =\beta_{C} \omega_{R} \phi_{C}^{2} \int_{\varphi_{0}}^{\pi-\varphi_{0}} \cos ^{2} \varphi(Q \sin \varphi-1)^{2 p} \mathrm{~d} \varphi+ \\
& +\beta_{C} \omega_{R} \phi_{C}^{2} \int_{\pi+\varphi_{0}}^{2 \pi-\varphi_{0}} \cos ^{2} \varphi(Q \sin \varphi+1)^{2 p} \mathrm{~d} \varphi,
\end{aligned}
$$

whose integration limits are the same as the original Kelvin-Voigt model. When $p=0$, $\sigma$ is given by Eq. [37), but there is no general formula for this integral as a function of $Q$ and $p$. As an example of its complexity, the formula for $\sigma$ when $p=1$ is shown below:

$$
\sigma=\frac{\beta_{C} \omega_{R} \phi_{C}^{2}}{4 \pi}\left(\left(Q^{2}+4\right)\left(\pi-2 \varphi_{0}\right)-\frac{2}{3} \cos \varphi_{0}\left(13 Q+2 \sin \varphi_{0}\right)\right)
$$

\section{Model tuning and analysis}

The frequency response for each impact model can be obtained by substituting the harmonic linearization coefficients found in Section 5.3 into the generic amplitudefrequency relationship, Eq. 27]. Then one can use a nonlinear least squares solver to fit the frequency response equations of each model to experimental data, such as the one from Fig. 22. Again, the experimental data is normalized using the fundamental linear natural frequency, $f_{n} \approx 7.6 \mathrm{~Hz}$, and grazing amplitude $\Delta_{G} \approx 1.6 \mathrm{~mm}$.

\subsection{Linear range}

Before defining appropriate values for the impact force model parameters, one should deal with the conversion gain $\gamma$ and structural damping ratio $\beta_{S}$, which are necessary to model both impacting and non-impacting vibrations. Fig. 7 7illustrates the normalized linear experimental frequency response together with its model approximations for $\gamma=0.195 \mathrm{~V}^{-1}$ and $\beta_{S}=14 \times 10^{-3}$. Despite been able to reproduce the resonance peak accurately, model predictions deteriorate outside the peak neighbourhood. That can be explained by the unmodeled dynamics of the subsystem shaker-platform. 


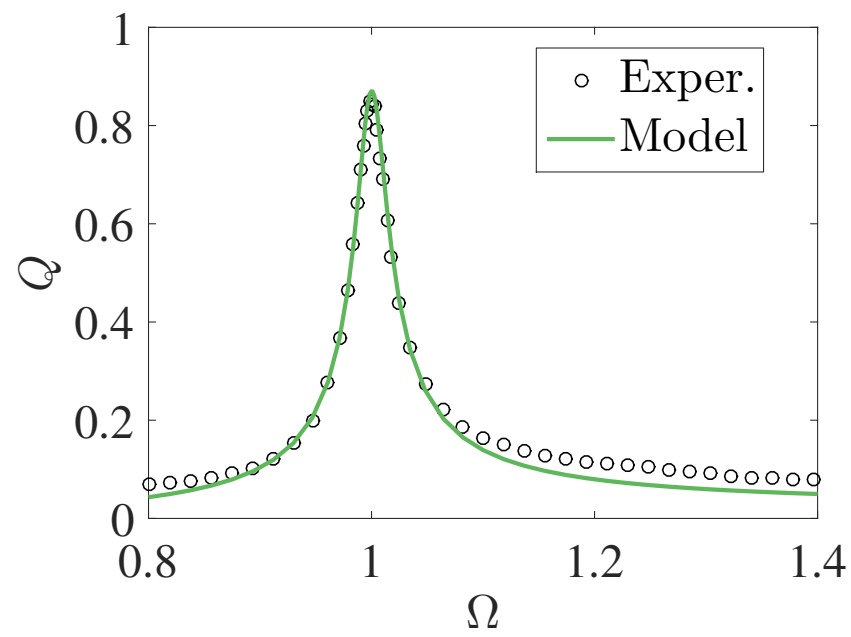

Figure 7: Linear frequency response, $\tilde{V}=0.125 \mathrm{~V}$.

For vibro-impact oscillations the excitation amplitude was chosen as $\tilde{V}=0.6 \mathrm{~V}$ in order to maximize the nonlinear frequency range without saturating the displacement sensors.

\subsection{Power function exponents}

The fitting of models with power function was made considering pairs of $1 \leq n \leq$ 10 and $0 \leq p \leq 10$ as constant while varying $\omega_{R}$ and $\beta_{C}$. This revealed some insights into the role of the power exponents on the frequency response, see Fig. 8 . For instance, by increasing $p$ the Power-law model response gets closer to the experimental upper folding point (Fig. $8 \mathrm{p}$ ). In this case, the fitted $\omega_{R}$ keeps almost constant, while $\beta_{C}$ decreases. By increasing $n$, as shown in Fig. 8 a the model response gets closer to both the upper and lower folding points, going further away from the upper one if $n$ grows too much. Also, by increasing $n$ the model response approaches the curvature of the bent peak. In this case, the fitted $\beta_{C}$ goes up, while $\omega_{R}$ goes down. This approach to the lower folding point and peak curvature for growing exponents can be explained by the fact that for small $n$ and $p$ the Power-law force is not negligible in the non-impacting region. For the MKV model, Fig. 8s shows that there are no significant changes for $1 \leq p \leq 5$, with the parameter $\omega_{R}$ remaining constant while $\beta_{C}$ grows. 

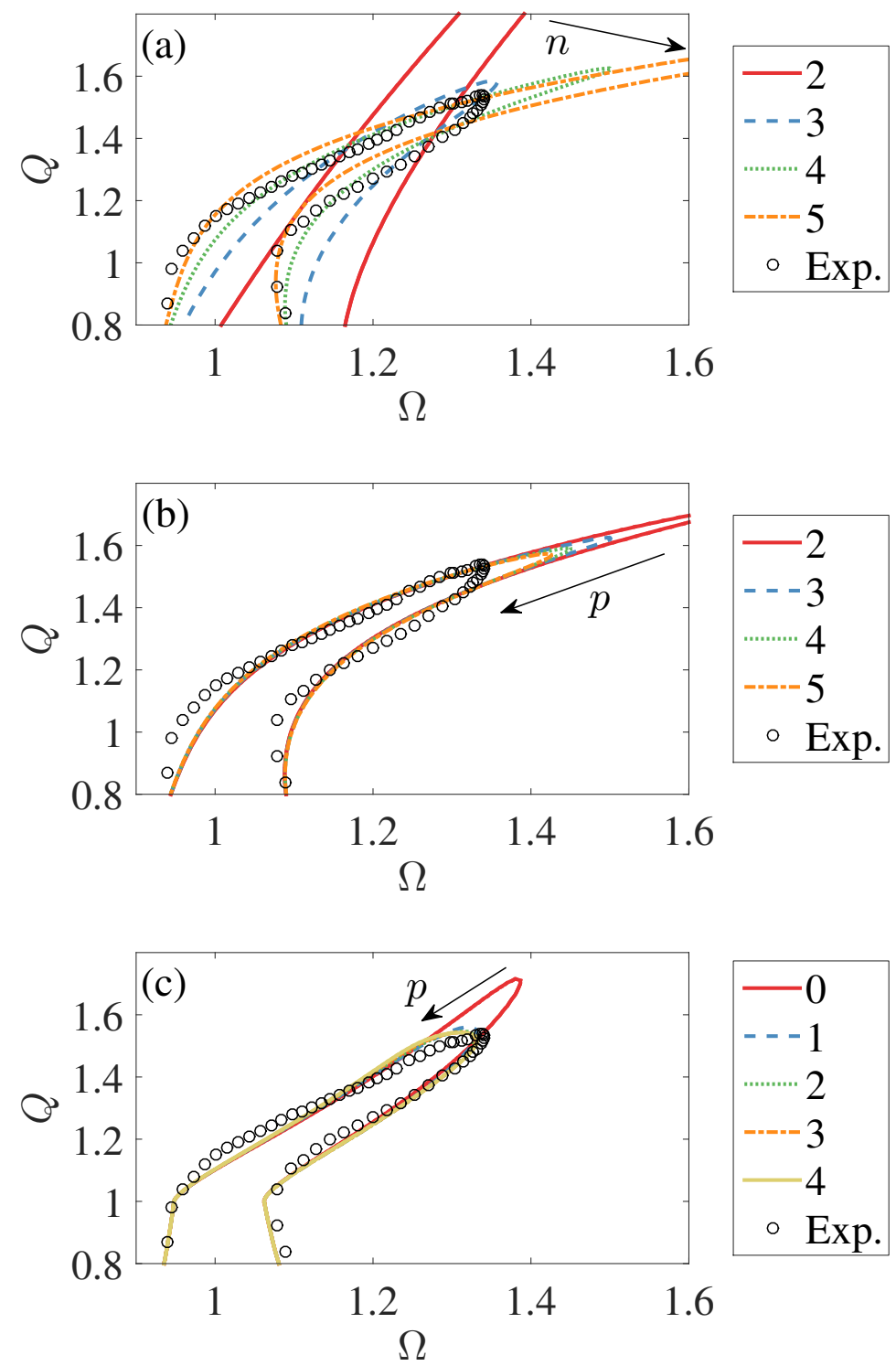

Figure 8: Comparison of power exponents on frequency response of the Power-law model (a) varying $n$ and $p=3$, (b) varying $p$ and $n=4$, and (c) varying $p$ for the Modified Kelvin-Voigt model. $\tilde{V}=0.6 \mathrm{~V}$. The arrows indicate how the frequency response changes as the value of each parameter increase.

Based on this analysis, the model parameters are chosen as the ones which led their models' frequency response closer to experimental data with the lower exponent. For the MKV model, the exponent $p=1$ is chosen as the one whose response is closer to 
Table 1: Fitted model parameters.

\begin{tabular}{ccccc}
\hline \multirow{2}{*}{ Model } & \multicolumn{4}{c}{ Parameter } \\
\cline { 2 - 5 } & $\omega_{R}$ & $\beta_{C}$ & $n$ & $p$ \\
\hline Kelvin-Voigt & 7.278 & 0.258 & - & - \\
\hline Power-law & 0.715 & 0.659 & 4 & 3 \\
\hline Mod. Kelvin-Voigt & 7.306 & 4.636 & - & 1 \\
\hline
\end{tabular}

experimental data. The fitted model parameters are shown in Table 1 were obtained by applying the same reasoning to all models. In this table one can see that the MKV model has lower exponent coefficient than the Power-law model. That can be explained by the fact that both KV models are only defined on contact areas, while the power function is defined also on the non-contact region and demands high order exponents to be negligible in this area.

\subsection{Model validation}

To check the validity of the assumptions made, the fitted values shown in Table 1 are used to obtain the frequency response of the system by numeric simulation of the single-DOF model from Eq. (10) together with the force models from Eqs. (13), (17) and 19 using a standard MATLAB ${ }^{\circledR}$ ODE solver ode 45 ( ) together with the option 'Events' to handle the transition between impacting and non-impacting regimes when necessary.

Looking at the results from the perturbation methods, numeric simulations and experiments shown in Fig. 9 one can see that all of the mentioned mathematical models give results reasonably close to experimental observations. Thus, the assumptions made appear adequate. Also, one can see that the modified Kelvin-Voigt model is able to predict the experimental behavior more precisely, capturing both fold points, while Kelvin-Voigt's classic model is further away from the upper folding point and the Power-law model is further away from both folding points. 

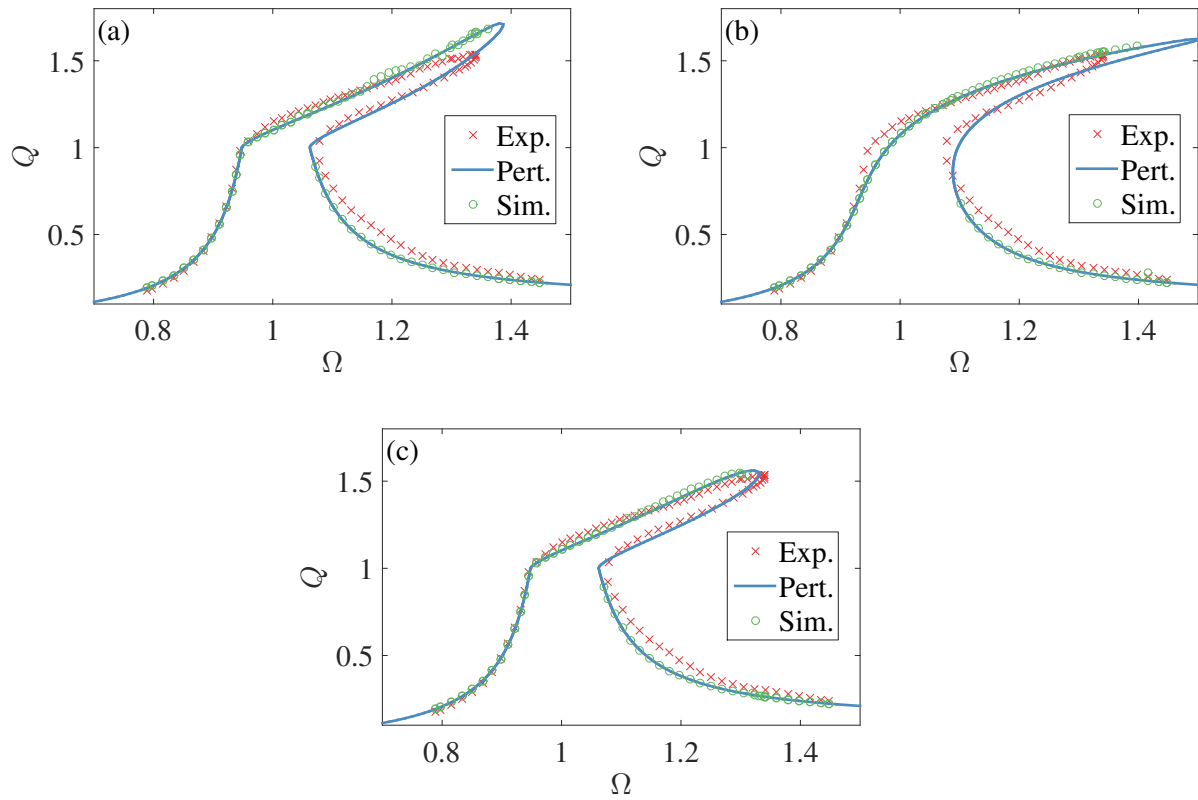

Figure 9: Comparison of frequency responses obtained using (a) the Kelvin-Voigt model (b) the Power-law model and (c) the Modified Kelvin-Voigt model. $\tilde{V}=0.6 \mathrm{~V}$.

\subsection{Parameter analysis}

After finding appropriate parameter values for the impact force models, one can analyze the individual effects of each parameter on the frequency response. That can expand one's knowledge about the different models, being used to design other vibroimpacting devices, which enhance or mitigate certain effects presented here such as the maximum response amplitude or the length and curvature of the bent peak.

Starting with Kelvin-Voigt's model, shown in Fig. 10, one can see that as expected, the peak curvature is very sensitive to $\omega_{R}$, with the peak length (hysteresis region) growing as $\omega_{R}$ increases. Also, by increasing the contact damping one can decrease the oscillation amplitude.

Similarly, for the Power-law impact model, shown in Fig. 11, one can see that the parameters related to the elastic force control the peak curvature and length, while the ones related to dissipative forces control the response amplitude. Increasing $\beta_{C}$ and $p$ causes the response amplitude to decrease. While increasing $\omega_{R}$ moves both folding 

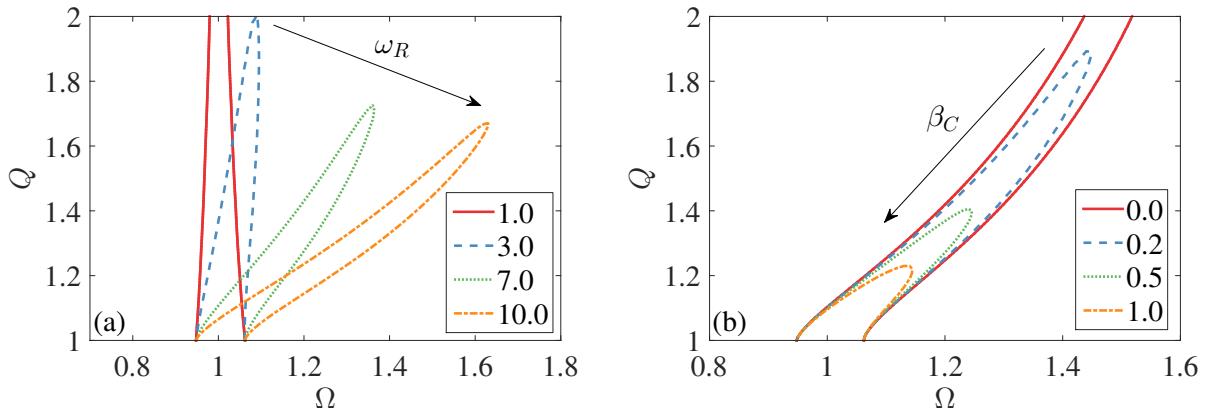

Figure 10: Parameter analysis of Kelvin-Voigt's model varying (a) $\omega_{R}$ and (b) $\beta_{C}$. The arrows indicate how the frequency response changes as the value of each parameter increase.
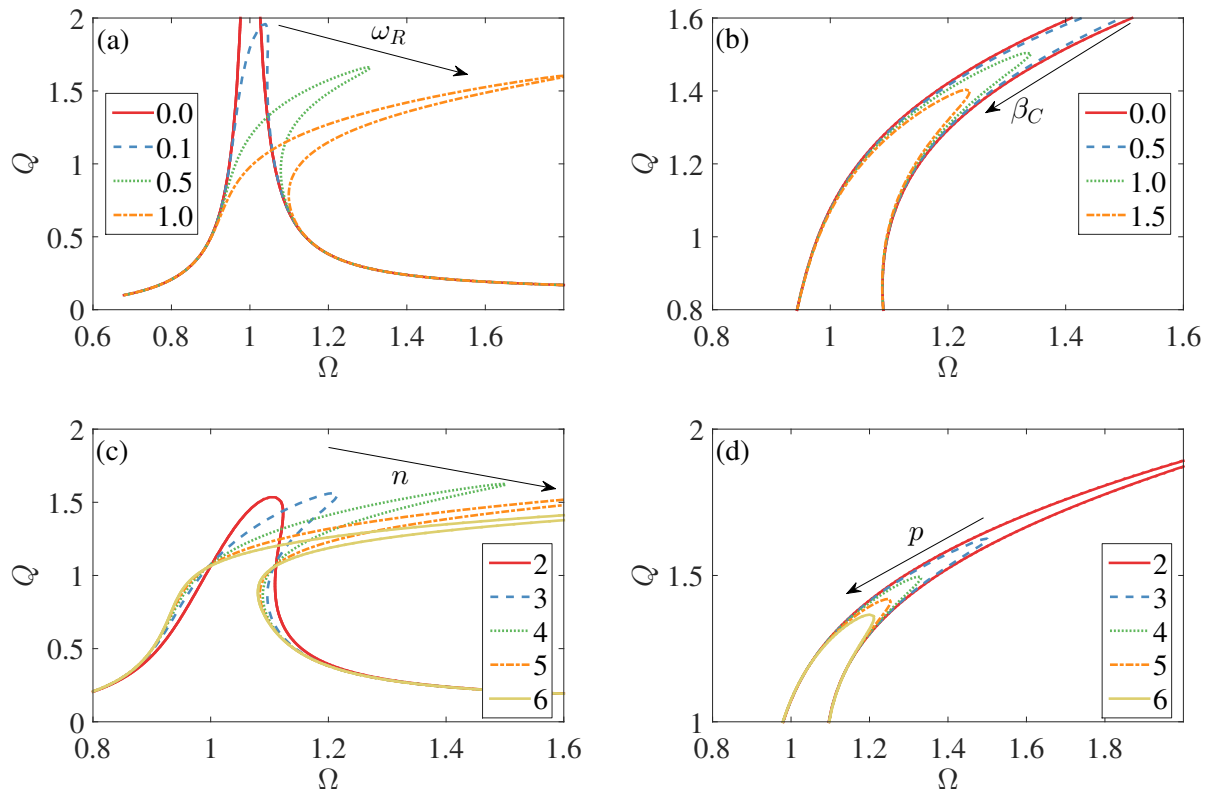

Figure 11: Parameter analysis of Power-law model varying (a) $\omega_{R}$, (b) $\beta_{C}$, (c) $n$ and (d) $p$. The arrows indicate how the frequency response changes as the value of each parameter increase.

points to the right, increasing $n$ moves the upper folding point to the right and the lower one to the left. Also, only the restoring force parameters have an influence on the non-impacting region of the frequency response.

The influence of the natural frequency ratio and contact damping ratio are the same for both versions of Kelvin-Voigt's model, see Figs. 10 and 12 a,b. However, different 

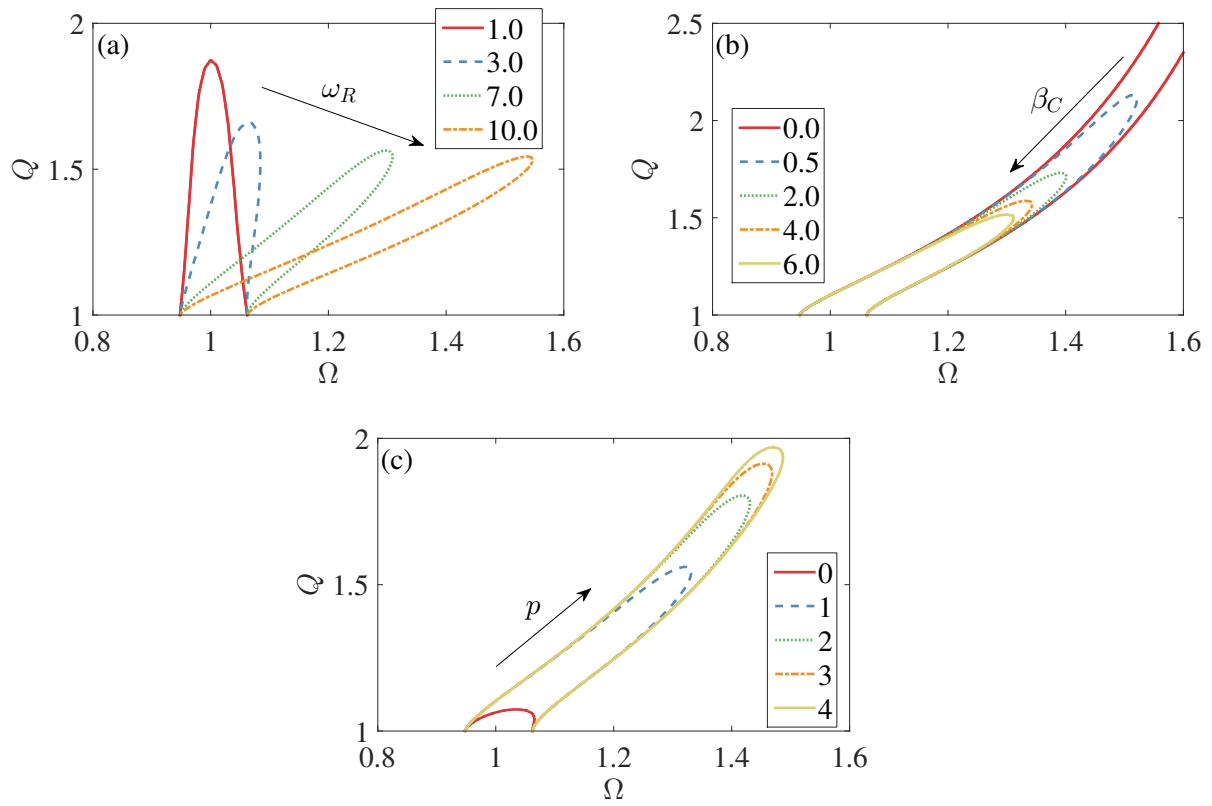

Figure 12: Parameter analysis of Modified Kelvin-Voigt model varying (a) $\omega_{R}$, (b) $\beta_{C}$ and (c) $p$. The arrows indicate how the frequency response changes as the value of each parameter increase.

from the Power-law model, the amplitude increases for higher values of $p$, see Fig. 12 . This happens because the dissipative force is proportional to $(q \pm 1)^{2 p}$, which, for lower exponents, is stronger in the neighborhood of impact, $Q \approx 1$. As $p$ increases the dissipative term becomes negligible for low impacting amplitudes. Also, it is worth to point out that despite the modified Kelvin-Voigt model being equivalent to its standard version for $p=0$, the frequency response on Fig. $12 \mathrm{r}$ is not equivalent to the one in Fig. $9 \mathrm{a}$ for the standard Kelvin-Voigt model because the contact damping has different numerical values.

\section{Conclusions}

The experimental behavior of an impacting forced lumped mass cantilever beam around its first resonance was modeled as a single degree of freedom oscillator. Harmonic decomposition of the frequency response of the experimental setup showed that higher harmonic components were negligible if compared with the fundamental one. 
The SDOF model was analyzed using Averaging and Harmonic Linearization to obtain frequency-amplitude relations. Numeric values for model parameters were obtained by fitting the nonlinear frequency response relationship to the experimental frequency response obtained using control-based continuation. After choosing the most appropriate model parameters, their role on the frequency response was also analyzed.

For all the impact models discussed in the present work, it was found that length and curvature of the bent peak are governed by the parameter $\omega_{R}$, defined as the natural frequency ratio, while the contact damping ratio controls the amplitude of vibration. For the Power-law model, while the exponent of the elastic term has an influence on both impacting and non-impacting regions of the frequency response, the damping exponent affects only the amplitude of oscillation of the impacting region. Also, the exponent of the dissipative term has opposite effects on MKV and power function models.

Experimental observations could be reproduced by all of the impact force models under analysis, with the modified Kelvin-Voigt model describing the experimental frequency response more accurately, predicting both fold points, while the other models fail to predict the upper fold point. The reason for that relies on the modified KV model being a combination of the other models, mixing their positive characteristics to obtain a physically more accurate model. Nevertheless, numeric simulations of the MKV model are more demanding than the continuous Power-law model, due to its non-smoothness with respect to displacement and velocity. Also, the lack of a general compact formula for the equivalent damping term, which has to be obtained for every power exponent, gives rise to complicated expressions.

The experimental setup could be modified in order to analyze systems whose mass displacement is directly limited by the stops, becoming a test bed for experimental validation of kinematic impact models together with nonsmooth transformations [8, 36], thus, making it possible to compare also to kinematically based vibro-impact models in terms of ease of use, applicability and reliability.

The discussion presented in this work can be applied to the analysis of other mechanical systems with non-rigid constraints and whose steady-state oscillations are single-periodic. However, for different parameter configurations, the considered models can produce other qualitative behaviors, such as quasiperiodicity and chaos, not 
discussed here.

\section{Acknowledgments}

The authors express their acknowledgment to CAPES through the Science Without Borders Program with the process number: 99999.013736/2013-02, which sponsored the elaboration of this paper.

\section{References}

[1] A. E. Kobrinskii, Dynamics of mechanisms with elastic connections and impact systems, Iliffe, London, 1969.

[2] S. W. Shaw, P. J. Holmes, A periodically forced piecewise linear oscillator, Journal of Sound and Vibration 90 (1) (1983) 129-155. doi:10.1016/ $0022-460 \times(83) 90407-8$.

[3] A. C. J. Luo, The mapping dynamics of periodic motions for a three-piecewise linear system under a periodic excitation, Journal of Sound and Vibration 283 (35) (2005) 723-748. doi:10.1016/j.jsv.2004.05.023

[4] K. H. Hunt, F. R. E. Crossley, Coefficient of Restitution Interpreted as Damping in Vibroimpact, Journal of Applied Mechanics 42 (2) (1975) 440. do i: $10.1115 /$ 1.3423596 .

[5] M. Machado, P. Moreira, P. Flores, H. M. Lankarani, Compliant contact force models in multibody dynamics: Evolution of the Hertz contact theory, Mechanism and Machine Theory 53 (2012) 99-121. doi:10.1016/j. mechmachtheory.2012.02.010.

[6] P. Flores, H. M. Lankarani, Dissipative contact force models, in: Contact Force Models for Multibody Dynamics, Springer International Publishing, 2016, pp. 27-52. doi:10.1007/978-3-319-30897-5_3 
[7] S. R. Bishop, M. G. Thompson, S. Foale, Prediction of period-1 impacts in a driven beam, Proceedings of the Royal Society of London A: Mathematical, Physical and Engineering Sciences 452 (1954) (1996) 2579-2592. doi: $10.1098 / r s p a .1996 .0137$

[8] J. J. Thomsen, A. Fidlin, Near-elastic vibro-impact analysis by discontinuous transformations and averaging, Journal of Sound and Vibration 311 (1-2) (2008) 386-407. doi:10.1016/j.jsv.2007.09.007.

[9] B. Blazejczyk-Okolewska, K. Czolczynski, T. Kapitaniak, Dynamics of a twodegree-of-freedom cantilever beam with impacts, Chaos, Solitons \& Fractals 40 (4) (2009) 1991-2006. doi:10.1016/j.chaos.2007.09.097

[10] O. V. Gendelman, Analytic treatment of a system with a vibro-impact nonlinear energy sink, Journal of Sound and Vibration 331 (21) (2012) 4599-4608. doi: $10.1016 / j \cdot j s v .2012 .05 .021$.

[11] L. N. Virgin, C. George, A. Kini, Experiments on a non-smoothly-forced oscillator, Physica D: Nonlinear Phenomena 313 (2015) 1-10. doi:10.1016/j. physd.2015.09.002.

[12] R. Ramírez, T. Pöschel, N. V. Brilliantov, T. Schwager, Coefficient of restitution of colliding viscoelastic spheres, Physical Review E 60 (4) (1999) 4465-4472. doi:10.1103/PhysRevE.60.4465

[13] M. A. Savi, S. Divenyi, L. F. P. Franca, H. I. Weber, Numerical and experimental investigations of the nonlinear dynamics and chaos in non-smooth systems, Journal of Sound and Vibration 301 (1-2) (2007) 59-73. doi:10.1016/j.jsv. 2006.09 .014

[14] M. Elmegård, B. Krauskopf, H. M. Osinga, J. Starke, J. J. Thomsen, Bifurcation analysis of a smoothed model of a forced impacting beam and comparison with an experiment, Nonlinear Dynamics 77 (3) (2014) 951-966. doi:10.1007/ s11071-014-1353-x 
[15] T. Kim, T. Rook, R. Singh, Effect of smoothening functions on the frequency response of an oscillator with clearance non-linearity, Journal of Sound and Vibration 263 (3) (2003) 665-678. doi:10.1016/S0022-460X (02) 01469-4.

[16] O. Janin, C. H. Lamarque, Comparison of several numerical methods for mechanical systems with impacts, International Journal for Numerical Methods in Engineering 51 (9) (2001) 1101-1132. doi:10.1002/nme.206.

[17] V. Acary, B. Brogliato, Numerical Methods for Nonsmooth Dynamical Systems, Vol. 35 of Lecture Notes in Applied and Computational Mechanics, 10 Springer Berlin Heidelberg, Berlin, Heidelberg, 2008. doi:10.1007/ 978-3-540-75392-6.

[18] L. Dieci, L. Lopez, A survey of numerical methods for IVPs of ODEs with discontinuous right-hand side, Journal of Computational and Applied Mathematics 236 (16) (2012) 3967-3991. doi:10.1016/j.cam.2012.02.011.

[19] S. Aoki, T. Watanabe, Forced Vibration of Continuous System with Unsymmetrical Collision Characteristics, Nonlinear Dynamics 17 (2) (1998) 141-157. doi:10.1023/A:1008207721738

[20] T. Kim, T. Rook, R. Singh, Effect of nonlinear impact damping on the frequency response of a torsional system with clearance, Journal of Sound and Vibration 281 (3-5) (2005) 995-1021. doi:10.1016/j.jsv.2004.02.038.

[21] A. Narimani, Frequency Response of a Piecewise Linear Vibration Isolator, Journal of Vibration and Control 10 (12) (2004) 1775-1794. doi:10.1177/ 1077546304044795 .

[22] M. S. M. Soliman, E. M. Abdel-Rahman, E. F. El-Saadany, R. R. Mansour, A wideband vibration-based energy harvester, Journal of Micromechanics and Microengineering 18 (11) (2008) 115021. doi:10.1088/0960-1317/18/ $11 / 115021$.

[23] S. Liu, Q. Cheng, D. Zhao, L. Feng, Theoretical modeling and analysis of twodegree-of-freedom piezoelectric energy harvester with stopper, Sensors and Ac- 
tuators A: Physical 245 (2016) 97-105. doi:10.1016/j.sna.2016.04. 060.

[24] K. Zou, S. Nagarajaiah, Study of a piecewise linear dynamic system with negative and positive stiffness, Communications in Nonlinear Science and Numerical Simulation 22 (1-3) (2015) 1084-1101. doi:10.1016/j.cnsns.2014. 08.016

[25] V. Zhuravlev, Equations of motion of mechanical systems with ideal onesided links, Journal of Applied Mathematics and Mechanics 42 (5) (1978) 839-847. doi:10.1016/0021-8928(78)90031-X

[26] A. P. Ivanov, Analytical methods in the theory of vibro-impact systems, Journal of 1. Applied Mathematics and Mechanics 57 (2) (1993) 221-236. doi:10.1016/ 0021-8928(93) 90050-V.

[27] V. N. Pilipchuk, Nonlinear Dynamics, Vol. 52 of Lecture Notes in Applied and Computational Mechanics, Springer Berlin Heidelberg, Berlin, Heidelberg, 2010. doi:10.1007/978-3-642-12799-1.

[28] F. C. Moon, S. W. Shaw, Chaotic vibrations of a beam with non-linear boundary conditions, International Journal of Non-Linear Mechanics 18 (6) (1983) 465477. doi:10.1016/0020-7462(83)90033-1.

[29] R. R. Aguiar, H. I. Weber, Mathematical modeling and experimental investigation of an embedded vibro-impact system, Nonlinear Dynamics 65 (3) (2011) 317334. doi:10.1007/s11071-010-9894-0.

[30] E. Bureau, F. Schilder, I. F. Santos, J. J. Thomsen, J. Starke, Experimental bifurcation analysis of an impact oscillator-Tuning a non-invasive control scheme,

1. Journal of Sound and Vibration 332 (22) (2013) 5883-5897. doi:10.1016/ j.jsv.2013.05.033.

[31] E. Bureau, F. Schilder, M. Elmegård, I. F. Santos, J. J. Thomsen, J. Starke, Experimental bifurcation analysis of an impact oscillator-Determining stability, 
Journal of Sound and Vibration 333 (21) (2014) 5464-5474. doi:10.1016/ j.jsv.2014.05.032.

[32] H. M. Lankarani, P. E. Nikravesh, A Contact Force Model With Hysteresis Damping for Impact Analysis of Multibody Systems, Journal of Mechanical Design 112 (3) (1990) 369. doi:10.1115/1.2912617

[33] V. I. Babitsky, Theory of Vibro-Impact Systems and Applications, Foundations of Engineering Mechanics, Springer Berlin Heidelberg, Berlin, Heidelberg, 1998. doi:10.1007/978-3-540-69635-3.

[34] A. H. Nayfeh, Perturbation Methods, Vol. 56 of Texts in Applied Mathematics, Wiley-VCH Verlag GmbH, 2000. doi:10.1002/9783527617609.

[35] J. J. Thomsen, Vibrations and Stability, 2nd Edition, Springer Berlin Heidelberg, 2003. doi:10.1007/978-3-662-10793-5.

[36] V. N. Pilipchuk, Closed-form solutions for oscillators with inelastic impacts, Journal of Sound and Vibration 359 (2015) 154-167. doi:10.1016/j.jsv. 2015.08 .023 